\title{
Observer Development for Automatic STEM Closed-Loop Control
}

\author{
A. Tejada*, A. J. den Dekker*, and S. W. van der Hoeven* \\ * DCSC, Delft University of Technology, Mekelweg 2628 CD, Delft, The Netherlands
}

It is of great scienti c and industrial interest to transform STEM microscopes into exible, unsupervised, quantitative nano-measuring tools. This requires, among other things, to automate several procedures that are currently executed manually based mostly on visual feedback (e.g., alignment, measuring particles, etc.). Such automation must take into account the time dependence of the microscopes internal processes. For instance, measuring the aberration coe cients from images takes a nite amount of time, which must be taken into consideration if high-speed aberration correction is needed. From this perspective, the microscopes become dynamical systems and their automation can be addressed with the tools and methods of control theory.

To the best of our knowledge, except for the related work in [1], this perspective is lacking in the literature. Thus, we aim to provide such perspective here by introducing a control-oriented STEM analysis framework. To illustrate these ideas, consider the task of controlling defocus. That is, the automation task, also known as the control objective, consists on keeping the instantaneous defocus value, $f(t), t \quad \mathbb{R}^{+}$, within a certain distance from a prescribed defocus value $f_{\text {ref }}$. In mathematical terms, $f_{\text {ref }} f(t)<$ for all $t>0$. This condition can be satis ed if an appropriate controller is added to the microscope objective lens (OL) subsystem, as shown in the simpli ed conceptual model in Figure 1. The model shows the main components of any practical STEM control system: a subsystem to be controlled (the OL electronics), a controller, and a mechanism to measure the variable to be controlled (also known as an observer).

As with most physical systems, the dynamics of the OL electronics can be represented by a set of (possibly) nonlinear di erential equations

$$
x(t)=h_{l}(x(t) u(t)) \quad f(t)=g_{l}(x(t) u(t))
$$

where $x(t)$ represents the internal state of the lens (e.g., current), $u(t)$ is the lens input (e.g., the voltage applied to it), $h_{l}$ is the state evolution function, and $g_{l}$ is the observation function. Then, the role of the controller is to stir the lens input to keep the size of $e(t)=f_{\text {ref }} f(t)$, the error between $f_{\text {ref }}$ and the measured defocus value $f(t)$, as small as possible. Adapting available techniques, a controller can be designed to ensure that $e(t)<$ for all $t>0$, satisfying the aforementioned control objective if $f(t) \quad f(t)$. The latter depends heavily on the quality of the observer. Also, the controller design depends on the exact nature of $h_{l}$ and $g_{l}$, which must be derived from physical principles or from system identi cation experiments (this also requires the use of a high quality observer).

The observer design varies depending on the application. As shown in Figure 1, the measurement of optical parameters in a STEM is generally done through image analysis. For instance, defocus can be measured through HAADF images [2], Ronchigrams [3], or di ractograms [4]. In all cases, the defocus is estimated from the features of an image $I(r t)$, which is a function of the object function $(r)$ and the microscope's transfer function $h(r \quad f(t))=\mathfrak{F}^{1} \exp (i(q))$, where $r$ is the spatial coordinate, $q$ is the spacial-frequency coordinate, $\mathfrak{F}$ is the Fourier transform, and $(q)$ is the aberration function (see [5] for details). The notation $h(r \quad f(t))$ indicates that 
$f(t)$ is the only time-varying parameter in the transfer function. Thus, the process of image formation together with the process of estimating the defocus from the images can be though of as a dynamical system with input $f(t)$ and output $f(t)$. The behavior of such system is characterized by the relation

$$
f(t)=g_{o}(f(t \quad))
$$

where $g_{o}$ is usually a linear function, and is the delay introduced by processing an image .

As an example, an observer was built using the di ractogram-based approach in [4]. Di ractograms can be acquired concurrently with STEM dark eld images, (potentially) allowing online simultaneous defocus measurement. A hundred focus series of amorphous specimens were simulated using the approach in [6], with underfocus ranging from 70nm to $200 \mathrm{~nm}$ and considering only the e ects of defocus and spherical aberration in the transfer function. A Matlab program was written to autonomously estimate the defocus from each individual image in the series. The results (see Figure 2) show that the observer's response varies minimally from series to series, and that the observer can be modeled as $f(t)=1027 \quad f(t \quad) \quad 381$, with $\quad 4$ seconds.

Research is ongoing to complete the observer characterization. The observer will be used in the near future to identify the lens model, $h_{l}$ and $g_{l}$, using a combination of rst principles modeling and system identi cation techniques. [7].

[1] H. Fujioca et al., Journal of Electron Microscopy 35 (1986) 215.

[2] N. Tanaka et al., Ultramicroscopy 78 (1999) 103.

[3] N. Delby et al., Journal of Electron Microscopy 50 (2001) 177.

[4] W. M. J. Coene and T. J. J. Denteneer, Ultramicroscopy 38 (1991) 225.

[5] L. Reimer, Transmission Electron Micrsocopy. Physics of Image Formation and Microanalysis, Springer Verlag, Berling Heidelberg, 1993.

[6] G. Y. Fan and J. M. Cowley, Ultramicroscopy 21 (1987) 125.

[7] This research was sponsored by the Condor project at FEI company, under the responsibilities of the Embedded Systems Institute (ESI). This project is partially supported by the Dutch Ministry of Economic A airs under the BSIK program.

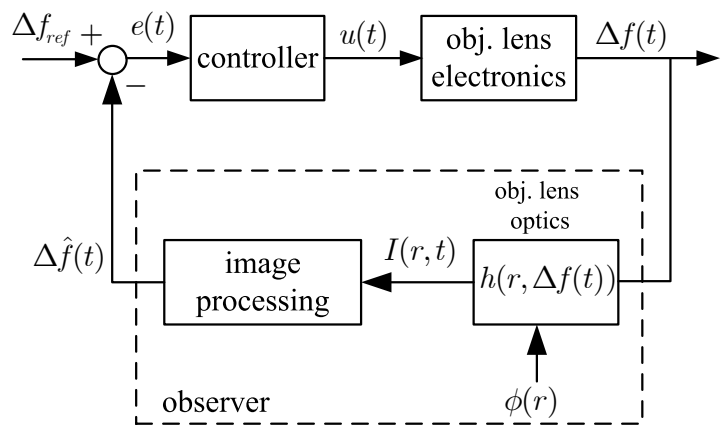

Fig. 1. Simpli ed STEM model.

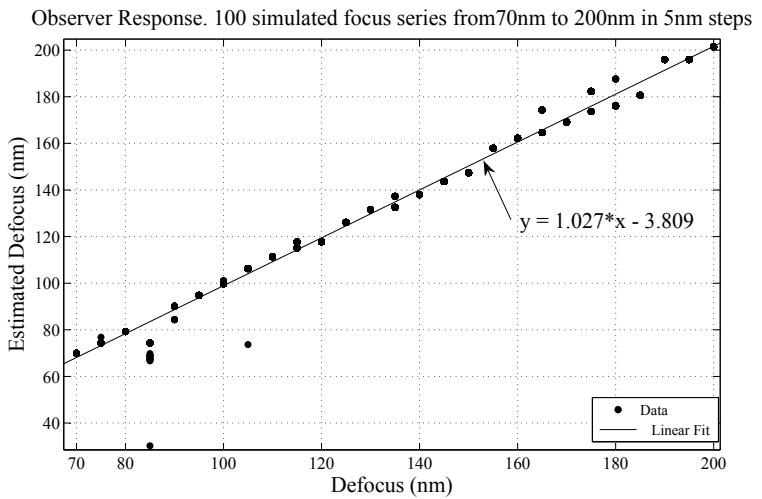

Fig. 2. One hundred overlayed responses of a di ractogram-based defocus observer. The outliers are software artifacts. 